\title{
Rinosinusitis frontal lateral oculta de rara presentación con signos de fístula palpebral, ectropión y lagoftalmos. Un informe de caso
}

\author{
Rare presentation occult lateral frontal rhinosinusitis with eyelid fistula, ectropion \\ and lagophthalmos. A case report
}

\begin{abstract}
Esteban Vergara-de la Rosa ${ }^{1,2,3 *}$, José Galvez-Olortegui ${ }^{4,5}$, Tomás Galvez-Olortegui ${ }^{4,6,7}$ y
Luis Concepción-Urteaga ${ }^{3,8}$

${ }^{1}$ Unidad de Investigación Clínica, Scientia Clinical and Epidemiological Research Institute, Trujillo; ${ }^{2}$ Servicio de Otorrinolaringología, Hospital Regional Docente de Trujillo, Trujillo; ${ }^{3}$ Facultad de Medicina, Universidad Nacional de Trujillo, Trujillo; ${ }^{4}$ Unidad Generadora de Evidencias y Vigilancia Epidemiológica, Scientia Clinical and Epidemiological Research Institute, Trujillo; ${ }^{5}$ Vicerrectorado de Investigación, Universidad Nacional Santiago Antúnez de Mayolo, Huaraz; ${ }^{6}$ Servicio de Oftalmología, Hospital Nacional Guillermo Almenara Irigoyen, Lima; ${ }^{7}$ sscuela de Posgrado, Universidad de San Martín de Porres, Lima; ${ }^{8}$ Servicio de Neumología, Hospital Regional Docente de Trujillo, Trujillo, Perú
\end{abstract}

\section{Resumen}

Se informa un inusual caso de un varón de 53 años, con fístula, ectropión y lagoftalmos derecho debido a rinosinusitis crónica frontal lateral. Dos años antes presentó tumefacción blanda y eritematosa en el epicanto interno. Un año y medio antes tuvo drenaje de secreción por fístula en el párpado superior, ectropión cicatrizal superomedial y lagoftamos ipsilateral, sin molestias otorrinolaringológicas ni alteración visual. El Servicio de Oftalmología realizó reparación quirúrgica mediante injerto de piel, sin mostrar mejoría, por lo que se solicitó resonancia magnética, que evidenció imagen indicativa de sinusitis frontal lateral derecha y fue transferido al Servicio de Otorrinolaringología. Se practicó una cirugía externa y endoscópica, que resolvió la afección sinusal, con buena evolución.

PALABRAS CLAVE: Fístula. Párpado. Sinusitis frontal. Ectropión. Lagoftalmos.

\begin{abstract}
We report an unusual case of a 53-year-old male with fistula, ectropion and lagophthalmos due to lateral frontal rhinosinusitis. Two years ago, he presented soft erythematous swelling at internal epicanthus. A year and a half before, he presented upper eyelid fistula secretion drainage, cicatricial ectropion and lagophthalmos. No otorhinolaryngological or visual discomfort was reported. Ophthalmology performed ectropion surgical repair using skin grafting, with no improvement. They requested magnetic resonance imaging which showed a suggestive image of frontal lateral sinusitis, being transferred to the Otorhinolaryngology service. External and endoscopic nasal surgery was performed, which resolved the sinus pathology with good evolution.
\end{abstract}

KEY WORDS: Fistula. Eyelids. Frontal sinusitis. Ectropion. Lagophthalmos.

\author{
Correspondencia: \\ *Esteban Vergara-de la Rosa \\ Scientia Clinical and Epidemiological Research Institute \\ Mz. G Lt. 22 Urb. \\ C.P. 13001, Vista Hermosa, Trujillo, Perú \\ E-mail: estebanvergara@ scientiaceri.com
}

Fecha de recepción: 01-11-2018

Fecha de aceptación: 02-05-2019

DOI: $10.24875 / C I R U .19000864$
Cir Cir. 2019;87(S1):77-80

Contents available at PubMed www.cirugiaycirujanos.com 


\section{Introducción}

La rinosinusitis frontal crónica es una anomalía frecuente que, en virtud de su proximidad a estructuras intracraneales y oftalmológicas, puede ocasionar complicaciones laterales. Las complicaciones orbitarias pueden afectar estructuras óseas o blandas y causar celulitis y abscesos, que a su vez pueden dar lugar a la formación de fístulas palpebrales si no se tratan'.

La fístula palpebral puede deberse, entre otras causas, a osteomielitis del hueso periorbitario y representar un signo de enfermedad paranasal subyacente ${ }^{2}$. Asimismo, la osteomielitis periorbital puede conducir al desarrollo de ectropión, eversión del borde del párpado inferior, por lo general clasificado involutivo, paralítico, mecánico y cicatrizal; esta última inusual ${ }^{3}$. En casos excepcionales, la presencia simultánea de lagoftalmos, producto de la retracción del párpado superior, puede incrementar los problemas de función ocular, además de producir una alteración estética². La presencia de alguno de los tres signos puede originarse por la falta de sospecha clínica de esta alteración poco frecuente y no reconocida por largos periodos de tiempo. Se han publicado pocos informes de casos o series de casos con uno de los signos ${ }^{1-3}$.

Se presenta el caso de un paciente con fístula palpebral, ectropión de párpado superior y lagoftalmos como signos de rara presentación de rinosinusitis frontal lateral, el primero notificado en la bibliografía consultada. La finalidad es reforzar la sospecha de una rinosinusitis frontal crónica lateral ante la presencia de estos signos oculares y disminuir el retraso diagnóstico para evitar complicaciones.

\section{Caso clínico}

Paciente masculino de 53 años que acude al servicio de otorrinolaringología del Hospital Regional Docente de Trujillo, en el norte de Perú. Es referido del Servicio de Oftalmología de otro hospital por presentar fístula y ectropión del párpado superior derecho acompañado de lagoftalmos ipsilateral. Dos años antes presentó tumefacción blanda, eritematosa y levemente dolorosa a nivel del epicanto interno derecho, acompañado de rinorrea derecha no maloliente. Un año y medio antes tuvo drenaje espontáneo de secreción purulenta en la tumefacción palpebral derecha, con formación posterior de fístula, secreción periódica acompañada de ectropión cicatrizal en el plano superomedial y lagoftalmos. No tenía antecedentes de

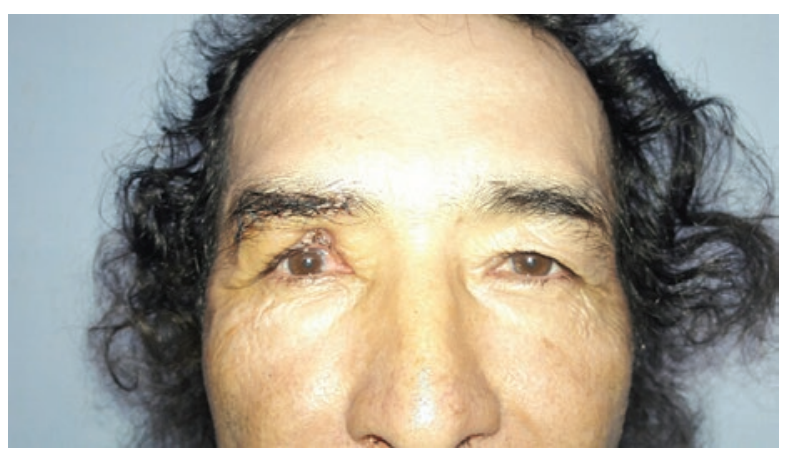

Figura 1. Vista frontal que muestra fístula y ectropión en el párpado superior derecho, con engrosamiento del tejido adyacente.

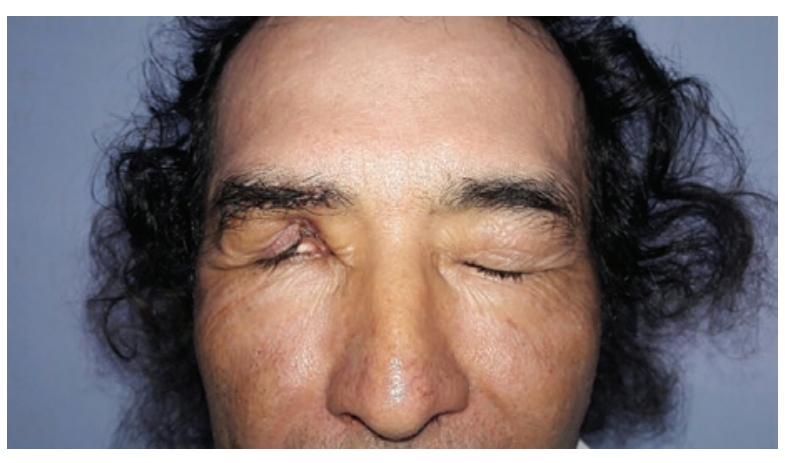

Figura 2. Vista frontal. Nótense el cierre de ojos y la presencia de lagoftamos derecho.

cefalea, alteración visual, hiposmia, enfermedad sistémica ni obstrucción nasal. El Servicio de Oftalmología realizó reparación quirúrgica del ectropión mediante injerto de piel con técnica de sutura, sin conseguir mejoría, por lo que se solicitó resonancia magnética (RM).

En la inspección se observaron ptosis derecha y leve disminución de la excursión del elevador del párpado, induración blanda e indolora superomedial del párpado superior, dolor a la percusión del reborde orbitario superior y lagoftamos con pequeña exposición corneal al cierre de ojos (Figs. 1 y 2). En la RM de globo ocular se reconoció una imagen consistente con rinosinusitis frontal lateral derecha con leve desplazamiento del globo ocular hacia abajo y la derecha (Fig. 3), objeto de indicación quirúrgica. Se realizó un abordaje quirúrgico combinado externo y endoscópico. En el abordaje externo se evidenció una fístula cutánea en párpado superior y cicatriz adherida a hueso subyacente, con presencia de pequeña lisis ósea a nivel anterior del seno frontal y salida de material purulento. De manera adicional se visualizó la presencia de un septo óseo dentro del seno frontal (Fig. 4), que impedía el drenaje de la secreción hacia el ostium frontal. Se practicó septeptomía medial de la celda lateral comprometida, hasta verificar 


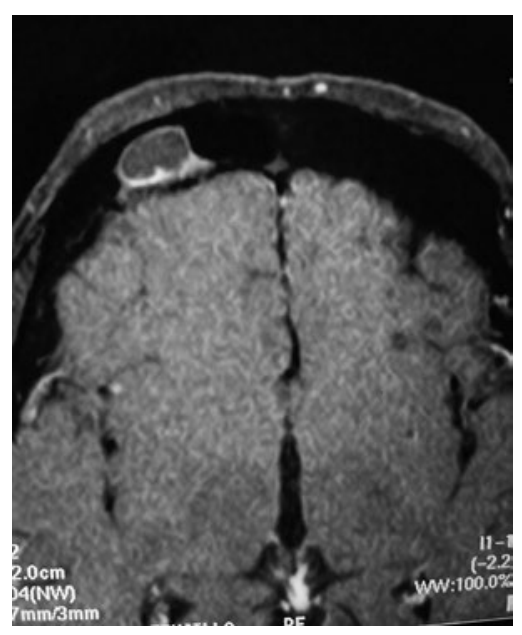

Figura 3. Corte axial prequirúrgico de la resonancia magnética. Obsérvese la presencia de imagen indicativa de secreción líquida frontal lateral derecha.

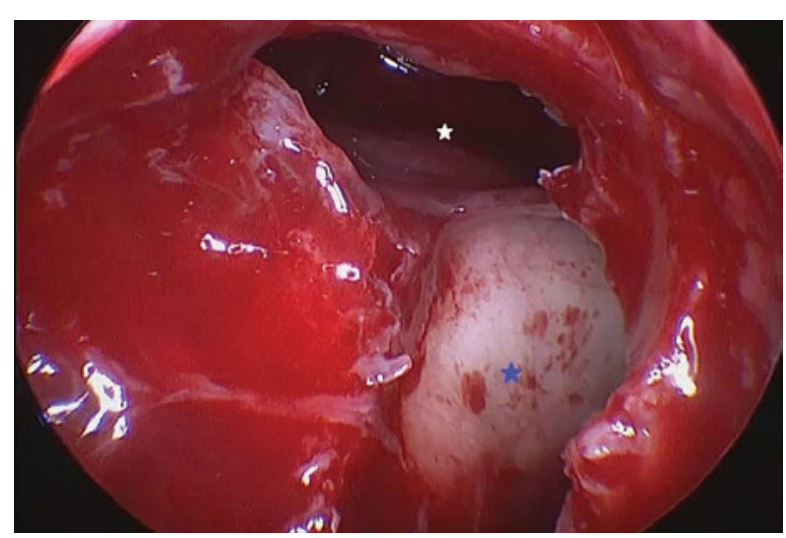

Figura 4. Vista endoscópica del seno frontal intraoperatoria por vía externa (telescopio de $30^{\circ}$ ). Nótese el septo rebajado que separa la celda lateral comprometida (proximal) de las celdas frontales mediales (distales) que comunican con el ostium frontal.

permeabilidad hacia el ostium frontal. De modo simultáneo se llevó a cabo un abordaje endoscópico del seno frontal que permitió reconocer un ostium permeable, por lo cual se introdujo una cánula de aspiración que se verificó de forma externa. El cultivo de la secreción fue negativo y el paciente presentó una evolución clínica favorable con tomografía de control sin alteraciones un año después de la intervención. El paciente padece aún retracción del párpado superior que requiere una operación oculoplástica complementaria.

\section{Discusión}

Las complicaciones reportadas de las rinosinusitis frontales crónicas tienen por lo general ubicación craneal u orbital debido a su localización anatómica y drenaje venoso; sin embargo, son infrecuentes las complicaciones de estructuras blandas y óseas adyacentes, como las fístulas, ectropión y lagoftalmo ${ }^{4}$. Se ha notificado que dichas complicaciones evitarían el compromiso intracraneal y orbitario por la presencia de la fístula, la que tendría una función de drenaje secundario ${ }^{4}$.

Existen pocos informes de tales complicaciones y sólo se ha identificado con frecuencia uno de los tres signos mencionados en este caso ${ }^{1-4}$. Uno de los factores comunes a estas complicaciones es su larga latencia (de meses a años) $)^{5}$ : se han comunicado casos que fluctúan entre 4 y 100 meses $^{3}$, un lapso similar al de este paciente y que incrementa la posibilidad de complicaciones craneales u orbitales. Además, se ha notificado la ausencia de antecedentes de traumatimos, cefalea, fiebre o síntomas marcados de rinosinusitis ${ }^{4}$, motivo por el que el diagnóstico final se establece tal vez meses o incluso años después del inicio de la enfermedad.

La cirugía endoscópica nasal es la elección para el tratamiento de la sinusitis crónica. Rossman, et al. ${ }^{2}$, en un estudio retrospectivo de ocho pacientes con fístula palpebral por rinosinusitis crónica, reportaron que siete pacientes se trataron de forma endoscópica y sólo uno mediante cirugía abierta. En este caso, la marcada lateralización de la lesión, la erosión ósea, y las publicaciones médicas consultadas ${ }^{6}$, orientaron el empleo de ambas técnicas.

En conclusión, se notifica un caso inusual de un paciente con rinosinusitis frontal complicado con fístula palpebral, ectropión y lagoftalmos y se enfatiza la relevancia de considerar la rinosinusitis crónica frontal subyacente como origen de estas complicaciones infrecuentes. Esto ayudará a los cirujanos maxilofaciales, otorrinolaringólogos y oftalmólogos a realizar un diagnóstico temprano, instituir un tratamiento oportuno y evitar complicaciones mayores que podrían poner en riesgo la vida del paciente.

\section{Financiamiento}

Los autores declaran no haber recibido ningún financiamiento para la realización de este trabajo. El manuscrito es elaboración original de los autores y todos los autores participaron de su elaboración.

\section{Conflicto de intereses}

Los autores declaran no tener ningún conflicto de intereses. 


\section{Responsabilidades éticas}

Protección de personas y animales. Los autores declaran que los procedimientos seguidos se ajustaron a las normas éticas del comité de experimentación humana responsable y de acuerdo con la Asociación Médica Mundial y la Declaración de Helsinki.

Confidencialidad de los datos. Los autores declaran que han seguido los protocolos de su centro de trabajo sobre la publicación de datos de pacientes.

Derecho a la privacidad y consentimiento informado. Los autores han obtenido el consentimiento informado de los pacientes o individuos referidos en este artículo. Este documento obra en poder del autor de correspondencia.

\section{Bibliografía}

1. Seyhan T, Ozerdem OR. Upper eyelid fistula caused by chronic frontal sinusitis. J Craniofac Surg. 2005;16(1):171-4.

2. Rossman D, Verity D, Lund V, Rose G. Eyelid fistula: a feature of occult sinus disease. Orbit. 2007;26(3):159-63.

3. Bandyopadhyay R, Chatterjee A, Banerjee S, Bandyopadhyay S, Mondol A. Frontal osteomyelitis presenting as upper eyelid ectropion: a cautionary tale. Saudi J Ophthalmol. 2015;29(3):238-41.

4. Toprak I, Demir C, Güngen S, Sarı T. Bilateral isolated sinopalpebral fistulas secondary to frontal sinusitis. Türk Oftalmoloji Dergisi. 2015;45(2):84-5.

5. Narendran N, Sandramouli S. A rare presentation of occult sinusitis: upper eyelid fistula. Ophthalmic Surg Lasers Imaging. 2010;42:1-3.

6. Hahn S, Palmer J, Purkey M, Kennedy D, Chiu A. Indications for external frontal sinus procedures for inflammatory sinus disease. Am J Rhinol Allergy. 2009;23(3):342-7. 